\title{
What is economics that physicists are mindful of it?
}

\author{
Sitabhra Sinha ${ }^{1, a}$, Anindya S. Chakrabarti ${ }^{2, b}$, and Manipushpak Mitra ${ }^{3, c}$ \\ 1 The institute of Mathematical Sciences, CIT Campus, Taramani, Chennai 600113, India \\ 2 Indian Institute of Management, Ahmedabad, Gujarat 380015, India \\ 3 Indian Statistical Institute, Kolkata, West Bengal 700108, India
}

Received 15 November 2016

Published online 22 December 2016

The genesis of this Discussion and Debate special issue of European Physical JournalSpecial Topics can be traced to discussions we had with several physicists and economists over the last few years that made us feel that this is an appropriate time to take stock of what the study of economic phenomena using the tools of physics has achieved and what it has failed to do so far. While several review papers have appeared in the recent past, these mostly focus on specific econophysics models and rarely consider broader questions about the utility and desirability of a physics-based approach for understanding economic phenomena. In particular, we were interested to know how physicists, as well as economists, view the strengths and weaknesses of econophysics vis-a-vis the conventional theoretical tools of economics. We believe that such a comparative discussion is extremely important for both subjects, but unfortunately this is rarely discussed at length in regular academic publications. We therefore thought of putting together a volume that will not be a mere technical review or compilation of research papers, but a more introspective assessment by practitioners in this interdisciplinary field.

We note that the previous year marked two decades from the time the term "econophysics" was coined, although physicists (or more generally, scientists using techniques borrowed from physics) have been working on economics problems even earlier. Indeed, it is also just about half a century since the publication of Mandelbrot's famous paper showing that price fluctuations do not follow a normal distribution as widely believed at the time, but rather a distribution having fat tails well-described by a power law. This observation has had far-reaching implications - not least being the realization among many that the range of phenomena forming the subject matter of statistical physics may also include economic systems. From the 1990s onwards there has been a virtual avalanche of publications in econophysics. These have mostly focused on properties of financial markets, complex economic networks, wealth \& income distribution, and strategic decision making.

A distinct character of the majority of such physics-based approaches has been the emphasis on uncovering "universal" phenomena, i.e., properties that are relatively

\footnotetext{
a e-mail: sitabhra@imsc.res.in

b e-mail: anindyac@iima.ac.in

c e-mail: mani@isical.ac.in
} 
invariant across systems. This emphasis on universality is easy to understand once one considers the history of statistical physics in the last century. The realization that phase transitions in systems as diverse as fluids, magnets and superconductors have features that are not only qualitatively, but quantitatively identical (depending on the dimensionality of the system) has led to one of the biggest advances in our understanding of the physical universe. It has culminated in a comprehensive theory of critical phenomena based on the renormalization group concept that explains why the behavior of systems in the neighborhood of their critical point becomes independent of their distinct micro-level features. Thus, a theory for describing aspects of the transition from a fluid into vapor can be successfully translated into one that helps in explaining features of the process by which the property of magnetization is lost on increasing the temperature, even though fluids and magnets are physically very different systems (e.g., involving different types of interactions between their constituent elements). Therefore, it is probably not surprising when physicists try to use a model originally developed to explain magnetism for understanding how markets are suddenly gripped by panic, although economists may find it very strange, to say the least.

Another valuable historical lesson that statistical physics has to offer to those seeking to extend it to economics concerns the importance of empirical data. Only by considering results from a large number of well-controlled experiments in a variety of systems did physicists became aware of the existence of universality in critical phenomena. Unsurprisingly therefore, much of the effort of scientists working in this inter-disciplinary field has been focused on financial markets, an area of economics where high-quality data is plentiful. The fortunate conjunction in the 1990s of the creation of large digital repositories of high-frequency trading statistics and the availability of cheap computational power to analyze such huge volumes of data, has been possibly the biggest driving force of econophysics. Much of the research work, therefore, has been and remains empirical in nature, which in turn has motivated the creation of parsimonious models for explaining the features discovered. The 'inverse cubic law' describing the distribution of fluctuations in stock prices or index movements is an example of a robust stylized fact established by physicists. Another very robust set of stylized facts goes under the name of Pareto laws for different macroeconomic quantities. Although this was proposed more than a century ago, the recent resurgence of interest in both the economics and econophysics literature owes an intellectual debt to the interdisciplinary work done on this topic over the last two decades. On the theoretical front, the encounter between physicists and economists have inspired work on agent based models, two prime examples being the minority game and asset exchange models. Last but not the least, the surge of interest in the analysis of large complex networks in the last decade has recently also made itself felt in the world of economics with more papers being published on different economic networks each year.

As with all other sciences, we realize that there are major limitations in the proposed tools and techniques used by econophysicists. The much coveted idea of universality may be the exception rather than the rule in economics and the social sciences. Another unanswered question is the applicability of the proposed paradigms to reality. Many of the models have been argued to be simplistic rather than simple. It has been pointed out by some that the criticisms that physicists make of economists can be also made about econophysics. For example, the charge that economists take their theories too seriously, often in disregard of empirical findings, may also be levelled at the attempts of physicists. In particular, there seems to be an absence of any clearcut demonstration of superiority of the econophysics models over standard economics models. 
Given that both the physics and economics approaches are highly mathematical and quantitative, one may well ask if there are any fundamental differences between how they treat the system under investigation. This may reduce to asking what is the principal distinction that sets apart economic from other classes of systems. We believe that the main difference is that the former is necessarily "forward-looking", in the sense that current actions are influenced by perception of future events. This perception may of course be influenced by what has happened in the past, but also by the biases and prejudices of the individual actors. How would this fact of the future (or rather the anticipation of it) being the driving force influence the behavior of a system? Would physics approaches provide novel perspectives for understanding how such systems work? It could well be that instead of there being a sharp distinction between economics and physics, there is a continuum of approaches lying closer to one discipline or the other.

We hope that the selection of articles in this volume will lead to a refreshing dialogue between the fields of economics and physics that will go some way towards addressing the above-mentioned issues. We have been fortunate in having a balanced representation of physicists and economists among our contributors. We hope that this will give a comprehensive picture of how the field has been viewed in various quarters and how it has evolved over the last couple of decades. Along with more introspective articles there are also technical papers that provide glimpses of new directions the field is taking. We look forward to a new decade of econophysics research that will hopefully see a more intimate engagement between the two scientific disciplines. 